\title{
Predicción de complicaciones pulmonares posoperatorias a través de pruebas de función pulmonar y pruebas cardiopulmonares en pacientes llevados a cirugía no torácica
}

\author{
Prediction of postoperative pulmonary complications \\ through pulmonary function tests and cadiopulmonary \\ tests in patients undergoing non-thoracic surgery
}

Alirio Rodrigo Bastidas¹, Yussef Diab², Freddy Chayanne ${ }^{3}$

\begin{abstract}
Postoperative pulmonary complications have a significant incidence and impact on morbidity and mortality in patients undergoing non thoracic surgery, hence the importance of identifying patients at risk, the role of pulmonary function tests and tools to take Preoperative measures to reduce complications. A search was conducted in databases from 2009 to 2018, finding that patient characteristics and type of surgery are the most important predictors for complications with a limited role of lung function testing and deployment option risk scales that help identify patients at risk.
\end{abstract}

\section{RESUMEN}

Las complicaciones pulmonares postoperatorias tienen una gran incidencia e impacto en la morbimortalidad de los pacientes llevados a cirugía no torácica, de ahí la importancia de identificar los pacientes en riesgo, el papel de las prue-

\section{Key words:}

Postoperative

complications,

lung,

postoperative period,

risk factors,

respiratory function

tests,

non-thoracic surgery

\section{Palabras clave:}

Complicaciones

posoperatorias,

pulmón,

período posoperatorio,

factores de riesgo, prue-

bas de función

respiratoria,

cirugía no torácica.

\footnotetext{
Maestría Epidemiología, Especialización en Neumología y Medicina interna, profesor de la Unidad de Investigación, Universidad de la Sabana. Neumología.

2 Magíster Telemedicina, Médico, Estudiante de Posgrado, Universidad de la Sabana, Anestesiología y Medicina Perioperatoria.

3 Médico, Estudiante de Posgrado, Universidad de la Sabana, Anestesiología y Medicina Perioperatoria.
}

Fecha de recepción: 16 de febrero de 2019

Fecha de aceptación: 15 de marzo de 2019

\section{ORCID}

https://orcid.org/0000 -0002-4656-7566

Correspondencia:

Aliro Rodrigo Bastidas

alirio.bastidas@clinicaunisabana.edu.co 
bas de función pulmonar y herramientas que permitan tomar medidas preoperatorias para disminuir las complicaciones. Se realizó una búsqueda en bases de datos seleccionando artículos con fechas desde 2009 a 2018, encontrando que las características de los pacientes y el tipo de cirugía son los predictores más importantes para complicaciones, con un papel limitado de las pruebas de función pulmonar y una opción de implementación de escalas de riesgo que ayudarían a identificar pacientes en riesgo.

\section{Introducción}

as complicaciones pulmonares postoperatorias (CPP) tienen una incidencia general reportada de $5-8 \%$, sin embargo, se ha descrito que la incidencia puede variar entre $5 \%$ al $70 \%$, principalmente por las diferentes definiciones empleadas de CPP. Se estima que la mortalidad durante la primera semana en el postoperatorio secundario a CPP se encuentra entre 8 a 24\%[1],[2],[3] y las CPP se han asociado con peores desenlaces postoperatorios, mayor estancia hospitalaria y consultas a urgencias[2]. Por tal motivo, la identificación temprana de pacientes con riesgo para desarrollar CPP permitiría al médico tratante instaurar estrategias perioperatorias que disminuyan la morbimortalidad. El objetivo de este estudio es revisar los cambios fisiopatológicos de la función pulmonar en pacientes bajo anestesia, la evaluación del riesgo de complicaciones pulmonares y las posibles medidas para disminuir la aparición de este tipo de complicaciones.

\section{Metodología}

Revisión no sistemática de la literatura en las bases de datos electrónicas PubMed, Cochrane, ScienceDirect, EBSCO, Ovid y Lilacs de artículos de 1 de enero de 2009 a 31 de diciembre de 2018, incluyendo ensayos clínicos controlados, estudios de cohorte, casos y controles, corte transversal, revisiones sistemáticas y metaanálisis limitándose por idioma castellano e inglés, incluyeron los estudios que relacionaban fisiología pulmonar bajo anestesia en pacientes con y sin patología pulmonar llevados a cirugía no cardiotorácica, pruebas de función pulmonar y de ejercicio incremental y no incremental en la evaluación y desenlaces posoperatorios, y utilización de reglas de predicción clínica en evaluación de pacientes, los artículos fueron revisados por todos los autores incluyéndose los Servicios de Anestesia y Neumología de la Clínica de la Universidad de la Sabana y Hospital Militar Central.

\section{Resultados}

Se revisaron en total 3.520 títulos y resúmenes ingresando a la revisión final 54 artículos, de los cuales se sintetizan los cambios fisiológicos asociados a los efectos anestésicos, pruebas de función pulmonar y pruebas de estrés en la predicción de complicaciones pulmonares posoperatorias (CPP) en cirugía no cardiotorácica.

\section{Efectos de la anestesia en la fisiología pulmonar}

Los cambios fisiológicos respiratorios secundarios a la anestesia se presentan tanto en la ventilación como en el intercambio gaseoso a nivel pulmonar. En la ventilación el volumen pulmonar en reposo disminuye de 0,8 a $1 \mathrm{~L}$ con el cambio de posición de bipedestación a decúbito supino, con la anestesia general este volumen pulmonar se reduce de 0,4 a $0,5 \mathrm{~L}$ adicionales[4]. Los anestésicos producen disminución de la capacidad residual funcional (CRF), explicada por el desplazamiento cefálico del diafragma, por una disminución del tono muscular tanto por efecto anestésico como por el tipo de cirugía. La disminución de los volúmenes pulmonares se evidencia en un $20 \%$ a un $40 \%$ en cirugías de abdomen superior, en comparación con un $2 \%-5 \%$ en las de abdomen inferior; estos efectos también se observan durante la cirugía laparoscópica, donde el neumoperitoneo ejerce presión mecánica sobre el diafragma[4]. En los pacientes llevados a cirugía abdominal laparoscopia existe un aumento del $\mathrm{CO}_{2}$ secundario al neumoperitoneo y a la rápida absorción de este gas al sistema circulatorio, permitiendo hipercapnia y cambios en la ventilación para normalizar los valores de estos gases[5],[6].

Goran y Lennart encontraron un aumento en la resistencia de las vías aéreas por una disminución en la capacidad residual funcional, descenso de la compliance pulmonar y disminución del diámetro de la vía aérea, que sumado a la administración de elevadas fracciones inspiradas de oxígeno, favorece la apari- 
ción de atelectasias de reabsorción en cerca de 90\% de los pacientes[4]. Todas estas alteraciones finalmente llevan a compromiso en la ventilación alveolar, de la relación ventilación perfusión y crean por otra parte focos susceptibles de infección que aumentan el riesgo de complicaciones pulmonares[4].

La relación ventilación perfusión (V/Q) se encuentra alterada debido al cambio en la distribución del flujo sanguíneo, desde las zonas dependientes a las zonas no dependientes del pulmón. Este cambio en la circulación redistribuye parte del flujo sanguíneo de los segmentos basales pulmonares hacia la regiones posteriores y apicales, generando un aumento del espacio muerto, principalmente en las zonas no dependientes e incrementando el shunt en las zonas dependientes. Normalmente estos cambios en la circulación se ven compensados por la vasoconstricción pulmonar hipóxica que redistribuye nuevamente el flujo sanguíneo de las regiones hipóxicas, a las que brinden mejor ventilación; sin embargo, durante la anestesia la mayoría de los agentes inhalados inhiben esta respuesta compensadora de la vasoconstricción pulmonar hipóxica, lo que conlleva a perpetuar las alteraciones V/Q[4],[7].

Las enfermedades pulmonares alteran aún más las respuestas fisiológicas y pueden disminuir la reserva ventilatoria, así como alterar aún más el intercambio de oxígeno según el tipo y gravedad de la misma. Los estudios paraclínicos utilizados para el diagnóstico y seguimiento de estas enfermedades podrían entonces, con la evaluación de la función pulmonar, servir como herramientas predictoras de complicaciones pulmonares, sin embargo, la evidencia disponible no recomienda el uso rutinario de las pruebas de función pulmonar previo, durante y posterior al acto anestésico en cirugía no cardiotorácica.

\section{Pruebas de función pulmonar y pruebas de estrés}

La espirometría, volúmenes pulmonares y análisis de la curva flujo volumen, proveen información de la mecánica respiratoria de los pacientes, mientras que los factores de transferencia y gases arteriales ayudan a evaluar la capacidad del parénquima pulmonar para el intercambio gaseoso. Los test de ejercicio sin prueba incremental (caminata de 6 minutos) e incremental (test de ejercicio cardiopulmonar) evalúan la respuesta dinámica de la función cardiaca y pulmonar durante la actividad física[8],[9]. El volumen espiratorio forzado $1\left(\mathrm{VEF}_{1}\right)$ y el $\mathrm{VEF}$, predicho postoperatorio son de utilidad como indicadores de complicaciones pulmonares postoperatorias posterior a una cirugía torácica y no están recomendados en cirugía no torácica, pero pueden ser de utilidad para optimizar el manejo de los pacientes con enfermedad respiratoria previo a la cirugía[8].

Las pruebas de función pulmonar pueden ser usadas en la evaluación preoperatoria de pacientes con factores riesgo, síntomas, sospecha clínica o diagnóstico de enfermedades pulmonares, que pueden llevar a complicaciones pulmonares postoperatoria en pacientes llevados a cirugía no torácica[8]. La importancia de estas pruebas son la identificación de pacientes con problemas respiratorios preexistentes y el determinar la respuesta al manejo médico previo a la cirugía[8].

\section{Espirometría y curva flujo volumen}

La espirometría evalúa las propiedades mecánicas del sistema respiratorio mediante la mediación de flujos y volúmenes espiratorios. Los valores normales varían dependiendo del género, raza, edad y talla, y puede ser usada para evaluar el patrón de la enfermedad pulmonar y establecer si hay respuesta a broncodilatadores, con la limitante que es un estudio dependiente del esfuerzo del paciente[10].

En pacientes sin enfermedad respiratoria las guías de la American College of Physicians recomiendan el no solicitar la espirometría de rutina para evaluar el riesgo de complicaciones pulmonares postoperatorias en pacientes llevados a cirugía. En un estudio reciente que evalúa la necesidad de espirometría en pacientes mayores de 60 años llevados a gastrectomía por laparoscopia, encontraron que la espirometría no era un estudio que identifica de forma fiable el riesgo de CPP en esta población quirúrgica[11],[12]. En pacientes mayores de 60 años llevados a gastrectomía por laparoscopia, la espirometría preoperatoria no fue confiable para predecir las CPP, ya sea como parámetros combinados o individuales, por lo cual, el estudio de Jin $\mathrm{H}$ et al., no apoya el uso rutinario de la espirometría para estratificar el riesgo de CPP en esta población quirúrgica[48]. Por otro lado, Tak Kyu Oh, In Sun PArk, Eunjeong y Hyo-Seok, encontraron en un estudio retrospectivo observacional de pacientes mayores de 60 años, fumadores y con historia de enfermedad pulmonar crónica, asociación entre la disminución de la CVF y la aparición de CPP en el contexto de cirugía abdominal laparoscópica, mientras $\mathrm{VEF}_{1}$ y la relación $V_{E F} /$ CVF no tuvieron relación alguna, por tal motivo consideran la espirometría como un método de estudio importante un mes previo al procedimiento quirúrgico[48]. En pacientes llevados a cirugía ortopédica, no se recomienda la espirometría de rutina, solo en pacientes con síntomas respiratorios inexplicables 
antes de someterse al procedimiento[49]. En el caso de corrección de escoliosis, la función pulmonar se deterioró en la escoliosis extremadamente grave y en aquellos pacientes con enfermedad pulmonar restrictiva severa[49].

Se encuentra indicada en pacientes con EPOC y asma que presentan síntomas clínicos que requieren manejo médico y en quienes se desconoce la necesidad de un manejo médico más agresivo preoperatorio para optimizar la función pulmonar[8]. Particularmente los pacientes con enfermedad pulmonar crónica llevados a neurocirugía y pacientes con patología neuromuscular bajo anestesia general: en quienes una CVF menor de $40 \%$ del predicho o presiones máximas inspiratorias y espiratorias debajo de 30 $\mathrm{cmH}_{2} \mathrm{O}$, son factores de riesgo para extubación fallida en el postoperatorio[8],[12],[13].

Sin embargo, es importante resaltar que el diagnóstico de asma y enfermedad pulmonar obstructiva crónica, se realiza a través de la espirometría, así como la clasificación de severidad que dirigirá un tratamiento específico, el cual debe ser recibido por el paciente durante el pre y posoperatorio[14],[15],[16].

\section{Difusión de monóxido de carbono}

La prueba de difusión de monóxido de carbono (CO) evalúa la eficiencia de la membrana alveolar, dado que el CO es rápidamente captado por la hemoglobina por lo que su transferencia está limitada solo por la difusión y una disminución en la transferencia de CO reflejaría alteraciones de la membrana alveolocapilar[8]. Los gases arteriales también indican anormalidades en el intercambio gaseoso en la presencia de hipoxemia o hipocapnia inexplicable, sin embargo, ni la hipoxemia ni la hipercapnia evaluados, mediante gasimetría arterial o difusión de CO, han sido identificados como predicadores de riesgo independientes para CPP[2],[8]. Según Ganai y Ferguson no existe información de estudios descriptivos en el uso de difusión de monóxido de carbono lo cual limita la interpretación para modelos de predicción de riesgo de esta prueba[21].

\section{Caminata de seis minutos}

El test consiste en recorrer un corredor de 30 metros hasta un máximo de seis minutos, en donde pueden descansar y repetir la prueba, se toma la mayor distancia recorrida del total de intentos[19]. Según Paisani et al., Hightower et al., y Smith et al., sugieren que pacientes con pobre tolerancia al ejercicio tienen incremento de desarrollar complicaciones posoperatorias en cirugía cardiotorácica y no cardiotorácica[18],[19],[20]. Por el contrario, en el estudio de
Paisani et al., no encontró una diferencia estadísticamente significativa en la realización del test de caminata de seis minutos y la presencia o no de CPP $29 \%$ vs $20,9 \%(p, 15)$ en pacientes llevados a cirugía abdominal superior[19]. Aun así, en la literatura no existe suficiente evidencia para dar una recomendación. En una cohorte prospectiva de pacientes llevados a cirugía no torácica y torácica, el test de caminata de seis minutos menor o igual a 325 metros, tuvo relación con la presentación de CPP en pacientes con alto riesgo[52]. En un estudio de cohorte prospectiva de pacientes llevados a trasplante hepático se pudo determinar el tiempo de isquemia frío y el test de caminata de seis minutos disminuido como predictor de CPP, el análisis de regresión logística mostró que la CPP eran menos probables cuando las distancias al caminar preoperatorias eran más largas (IC de 95\%) la probabilidad se redujo a $0,589(0,357-0,971)$ por cada 50 m de caminata $(p=0,03)[53]$.

\section{Test de ejercicio cardiovascular}

El test de ejercicio cardiovascular es un método objetivo no invasivo para evaluar la capacidad funcional o física de una persona, el cual integra oxígeno espirado, concentraciones de dióxido de carbono, medición de flujo ventilatorio, captación de oxígeno y producción de dióxido de carbono[16],[17], lo que permite determinar la habilidad de la capacidad fisiológica individual, para responder a las demandas metabólicas del estrés quirúrgico[18],[19]. Es considerado un método objetivo no invasivo que permite evaluar la respuesta del corazón, los pulmones y el sistema musculoesquelético ante el ejercicio, se basa en la medición de producción de dióxido de carbono $\left(\mathrm{CO}_{2}\right)$ y consumo de oxígeno $\left(\mathrm{O}_{2}\right)$, con lo que se puede calcular el consumo máximo de oxígeno y el umbral anaeróbico[8],[18].

En la cohorte de 116 pacientes de Snowden et al, se ha encontrado relación entre el test de ejercicio cardiovascular dado por un umbral aerobio $<10,1 \mathrm{ml} /$ $\mathrm{kg} / \mathrm{min}$ en cirugía abdominal mayor y la presencia de mayor riesgo de morbilidad[22]. West et al, en dos estudios de cirugía de colon y cirugía de recto, también halló relación en el aumento de morbilidad al encontrarse un umbral aerobio bajo $<10,1 \mathrm{ml} / \mathrm{kg} / \mathrm{min}$ - 10,6 ml/kg/min respectivamente[23],[24]. Por otro lado, Hartley et al, encontró con 415 pacientes llevados a cirugía abdominal mayor secundario a aneurisma de aorta abdominal, un relación del umbral aerobio $>10,1 \mathrm{ml} / \mathrm{kg} / \mathrm{min}$ y el aumento de la mortalidad[25]. Moyes et al, encontró relación mortalidad y un umbral aerobio < 9 en un estudio con 108 pacientes llevados a cirugía gastrointestinal superior[26]. Es 
claro resaltar, que en la presente revisión de Levett y Grocott, consideran que los resultados de asociación entre el umbral aerobio y la morbimortalidad posoperatoria puede ser subestimada dada que los diferentes estudios no fueron cegados a los resultados del test de ejercicio cardiopulmonar[17].

Carlisle et al, han encontrado un mejor valor predictor de mortalidad en cirugía de aneurisma aórtico abdominal a través del test de ejercicio cardiopulmonar y el índice de Lee[27]. Snowden et al, hallaron mayor predicción de morbilidad posoperatoria con el uso del índice de VASI y el test de ejercicio cardiopulmonar[16],[22].

El test de ejercicio cardiopulmonar ha permitido identificar pacientes de alto riesgo dado la previsión de morbilidad y mortalidad posoperatoria. Sin embargo, difiere entre los tipos de cirugía encontrando el umbral de aerobio como el predictor óptimo de alto riesgo para cirugía abdominal mayor, pico de $\mathrm{VO}_{2}$ para cirugía bariátrica y $\mathrm{Ve} / \mathrm{VCO}_{2}$ para cirugía de aneurisma aórtico abdominal[19].

En pacientes con alto riesgo se considera solicitar estas pruebas de función pulmonar según la Société de pneumologie de langue francaise en la valoración preoperatoria, sin embargo, algunos estudios clínicos indican que las pruebas de función pulmonar (PFP) predicen en menor proporción a los pacientes con alto riesgo de complicaciones pulmonares postoperatoria después de una cirugía no cardiotorácica con respecto a la historia clínica y el examen físico[28]. Se han descrito dos indicaciones para las PFP en el ámbito preoperatorio, la primera es la utilidad en determinar en los pacientes con enfermedad pulmonar obstructiva crónica y asma con síntomas clínicos, el examen clínico no permite identificar si el paciente está en su estado basal o si la obstrucción puede ser reducida con un tratamiento más agresivo preoperatorio que permita optimizar la función pulmonar[28]. La segunda indicación son los pacientes con disnea en reposo o con ejercicio leve sin historia clínica de patología pulmonar que explique esa pobre tolerancia al ejercicio, en este contexto las PFP permiten diferenciar patología cardiaca de enfermedad pulmonar primaria susceptible de ser manejada previo a la cirugía[28].

En los pacientes que se encontraban programados para cirugía bariátrica abierta, los test de función pulmonar eran predictores para determinar riesgo de complicaciones pulmonares, sin embargo, las complicaciones pulmonares no son una causa frecuente de mortalidad en estos pacientes[29],[30]. Según el estudio de Clavelina-Gaytán et al, encontró en pacientes con obesidad que presentan síndrome de apnea obstructiva del sueño llevados a cirugía bariátrica, una al- teración de los valores de espirometría preoperatoria como predictor de complicaciones pulmonares posoperatorias[30].

En conclusión, no hay evidencia que soporte el uso de rutina de las pruebas de función pulmonar para los pacientes llevados a cirugía no cardiotorácica, dado que la historia clínica y el examen físico es igual de efectivo en identificar esos pacientes en riesgo para complicaciones pulmonares postoperatorias[8],[31],[32].

\section{Enfermedades pulmonares en valoración preoperatoria}

La infección respiratoria aguda como el resfriado común, la nasofaringitis son infecciones virales que generan un mayor riesgo de laringoespasmo y broncoespasmo en niños así como acúmulo de secreciones. Por lo cual se recomienda posponer el procedimiento quirúrgico seis semanas después del episodio dado que se espera la resolución de la hiperreactividad de la vía aérea[14],[33]. En pacientes con comorbilidades dadas por asma y enfermedad pulmonar obstructiva crónica, se sugiere esperar hasta que se encuentre asintomático[14],[33].

En pacientes con asma, caracterizados por presentar obstrucción crónica de la vía aérea, obstrucción temporal del flujo espiratorio y reactividad de la mucosa bronquial, se considera deben presentar la clasificación de la gravedad de la enfermedad basado en una encuesta de síntomas, exacerbación, necesidad de rescate con inhaladores, interferencia con actividad física y pruebas de función pulmonar[35]. Sin embargo, el uso de pruebas de función pulmonar no ha modificado el manejo de los pacientes[35]. Así mismo, el manejo broncodilatador debe continuar antes y después del procedimiento quirúrgico; incluso en pacientes con presencia de sibilancias en el perioperatorio, se recomienda el uso de inhalador de acción corta e iniciar la inducción una vez las sibilancias se hayan resuelto[35]. De igual manera, el uso de propofol, genera efectos broncodilatadores, así como la ketamina produce relajación muscular lisa[35]. Medicamentos que inducen liberación de histamina como la morfina, meperidina y succinilcolina deben ser evitados a menos que sean necesarios en una emergencia[14].

El paciente con tabaquismo presenta 5 a 7 veces mayor riesgo de complicaciones pulmonares que los no fumadores, secundarias a disminución de la función mucociliar, hiperreactividad de la vía aérea, reducción de la función inmune y el riesgo del desarrollo de la enfermedad pulmonar obstructiva 
crónica[14],[36]. En cirugía programada el tabaquismo debe detenerse cuatro semanas antes del procedimiento, una suspensión abrupta puede producir aumento en la producción de esputo, ansiedad, inquietud, trastorno del sueño[14],[36]. El manejo del paciente en el perioperatorio debe similar al paciente con asma[14],[36].

En el paciente con enfermedad pulmonar obstructiva crónica debe ser considerada dado la presencia de hipoxemia e hipercapnia crónica, que puede llevar a acidosis respiratoria, hipertensión pulmonar, eritrocitosis y cor pulmonale[35]. Es recomendable determinar la severidad de la enfermedad, continuar el manejo farmacológico bajo el seguimiento de neumología [14]. En el perioperatorio, en los pacientes con bulas enfisematosas debe ser evitado el manejo con óxido nítrico dado el riesgo de ruptura, deben recibir oxígeno suplementario titulado, evitar exceso de oxígeno en pacientes con estado avanzado de enfermedad dado la alteración de la hipercapnia basal generando episodios de bradipnea[14]. El uso de opioides debe ser manejado con cautela dado el riesgo de depresión respiratoria que disminuye la actividad respiratoria hipercápnica que produce depresión respiratoria prolongada[14],[34],37].

\section{Complicaciones pulmonares y evaluación de riesgo}

Las complicaciones pulmonares posoperatorias tienen una frecuencia aproximada de $7 \%$ y puede variar entre el 5 a $70 \%$ dependiendo de la definición[39]. Se pueden dividir en complicaciones menores como atelectasias, broncoespasmo, traqueobronquitis a complicaciones severas que incluyen neumonía, exacerbación de EPOC, neumotórax o falla respiratoria aguda, que puede requerir reintubación o ventilación mecánica por más de 48 horas[38],[39].

Las causas de las complicaciones posoperatorias son multifactoriales y se pueden clasificar en implicaciones preoperatorias, intraoperatorias y posoperatorias[39]. Dentro de las preoperatorias se encuentran las condiciones del paciente, la capacidad función pulmonar y comorbilidades[38]. Las intraoperatorias son relativas al procedimiento quirúrgico, tipo de anestesia y el tipo cirugía electiva o de urgencia[38]. Los posoperatorios se relacionan cambios fisiológicos pulmonares posoperatorios como hipoventilación por anestesia, lesión de los músculos respiratorios, limitación del esfuerzo respiratorio por dolor, posiciones del paciente durante la recuperación[39]. Puede existir una reducción de $30 \%$ de la capacidad residual funcional y una reducción de $60 \%$ de la capacidad vital forzada que puede persistir por más de una semana después de una cirugía[39].

Los factores relacionados con la cirugía son aquellos que contribuyen de gran manera a la aparición de complicaciones pulmonares, siendo el sitio quirúrgico el mayor determinante[38]. La cirugía torácica, abdominal superior o de aorta abdominal tiene relación a presentar compromiso de los músculos respiratorios y el diafragma[38],[40]. Además, la duración de la cirugía y el tipo de anestesia con los bloqueos neuromusculares, contribuyen a las complicaciones pulmonares porque alteran los esfuerzos respiratorios del paciente[39].

En cuanto a los factores relacionados al paciente se puede describir que la edad, el estado mental y funcional, y las comorbilidades evaluadas por la clasificación de la Sociedad Americana de Anestesiología ASA como clase II o mayor y pacientes mayores de 65 años son factores de riesgo para complicaciones posoperatorias (CPO)[38]. Se han identificado comorbilidades de los pacientes con mayor relación a desencadenar CPO, pacientes fumadores, asmáticos, con EPOC, SAOS, hipertensión pulmonar, falla cardiaca y disfunción renal[41],[42].

Teniendo en cuenta los diferentes factores que pueden determinar las COP, se han descrito escalas de riesgo que incluyen Canet et al. 2010 - complicaciones pulmonares ARISCAT, Arozullah et al, 2001 Índice de riesgo pulmonar, Johnson et al, 2007. Índice de riesgo de falla respiratoria[42],[43],[44],,[45].

Cleva et al, encontró en un estudio de 60 pacientes llevados a cirugía abdominal, un alto riesgo de desarrollar complicaciones pulmonares, particularmente una disminución significativa del volumen espiratorio forzado en un segundo $\left(\mathrm{FEV}_{1}\right)$ y la capacidad vital forzada (FVC), aproximadamente de un 65 a 70\%[46].

En cirugías abdominales se ha encontrado alteración de las funciones pulmonares durante el posoperatorio en una revisión sistemática de Bablekos et al, se encontró una diferencia en la capacidad vital forzada entre colecistectomía laparoscópica $77,6 \%$ (73-80) y colecistectomía abierta 55,4\% (50-64) p $<0,001$, el volumen espiratorio forzado en un segundo $76 \%(72,2-81)$ vs $52,5(50-56,7) p<0,001$ y flujo espiratorio medido $25-75 \%$ con un valor de $78,8 \%(68,8-80,9)$ para colecistectomía laparoscópica vs $60 \%(56,1-66,1)$ para colecistectomía abierta $p$ $0,005[47]$. Existe un cambio en la presión transdiafragmática como resultado de la translocación del impulso respiratorio desde el diafragma a otros músculos asociados a la respiración lo cual genera una disminución del ancho de las oscilaciones diafragmáticas generando una restricción del esfuerzo espiratorio en cirugía abdominal superior[47]. 


\section{Discusión}

En la predicción de CPP en pacientes llevados a cirugía no cardiotorácica, la literatura considera la espirometría como un método que no debe realizarse de rutina; los pacientes que presentan enfermedades pulmonares crónicas de alto riesgo, debe considerarse su realización. De igual manera en pacientes llevados a cirugía ortopédica mayor y corrección de escoliosis, los pacientes de alto riesgo se benefician de una espirometría en la determinación de CPP. En cuanto a cirugía abdominal laparoscópica y mayor, existen estudios controversiales que no permiten concluir la necesidad de realizarse.
En cuanto al test de caminata de seis minutos, algunos estudios descriptivos determinan una relación inversamente proporcional entre distancia en metros recorridas y a la incidencia de CPP. Sin embargo, en la literatura no existe suficiente evidencia para dar una recomendación.

El test de ejercicio cardiovascular ha encontrado el aumento de la morbilidad y mortalidad posoperatoria en pacientes llevados a cirugía abdominal mayor con un umbral aerobio bajo $<10,1 \mathrm{ml} / \mathrm{kg} / \mathrm{min}$.

En conclusión, aun se necesitan estudios que puedan determinar la predicción de CPP en cirugía no cardiotorácica, teniendo en cuenta su alta incidencia de presentación en pacientes de alto riesgo y en cirugías mayores.

\section{Referencias}

1. Thanavaro JL, Foner BJ. Postoperative pulmonary complications: reducing risks for noncardiac surgery. Nurse Pract. 2013 Jul;38(7):38-47. https://doi.org/10.1097/01. NPR.0000431179.49311.0b PMID:23728493

2. Lakshminarasimhachar A, Smetana GW. Preoperative Evaluation: Estimation of Pulmonary Risk. Anesthesiol Clin. 2016 Mar;34(1):71-88. https://doi.org/10.1016/j. anclin.2015.10.007 PMID:26927740

3. Gallart L, Canet J. Post-operative pulmonary complications: understanding definitions and risk assessment. Best Pract Res Clin Anaesthesiol. 2015 Sep;29(3):315-30. https://doi. org/10.1016/j.bpa.2015.10.004 PMID:26643097

4. Goran H,Lennart E. Effects of anesthesia on the respiratory system. Best Practice \& Research Clinical Anaesthesiology 2015;29:273e284

5. Cleva, R, Assumpccao, M. Sasaya F, Chaves N, Santo M, Flo C, Lunardi A. Filho W. Correlation between intra-abdominal pres- sure and pulmonary volumes after superior and inferior abdominal surgery. Clinics. 2014; 69 (7:483-486. https://doi. org/10.6061/clinics/2014(07)07.

6. Karlmar A, Foubert $L$, Hendrickx J, Mottrie A, Absalom A, Mortier $\mathrm{E}$, et al. Influence of steep trendelenburg position and $\mathrm{CO} 2$ pneumoperitoneum on cariovascular, cerebrovascular and respiratory Homeostasis during robotic prostatectomy. Br J Anaesth. 2010;104:403-9.

7. Levett DZ, Grocott MP. Cardiopulmonary exercise testing for risk prediction in major abdominal surgery. Anesthesiol Clin. 2015 Mar;33(1):116. https://doi.org/10.1016/j. anclin.2014.11.001 PMID:25701925

8. Aubrey W, Saravanan P. Tests of pulmonary function before surgery. Anaesth Intensive Care Med. 2011;12(12):53941. https://doi.org/10.1016/j. mpaic.2011.09.012.

9. Zraier $\mathrm{S}$, Haouache $\mathrm{H}$, Dhonneur $\mathrm{G}$. Which preoperative respiratory evaluation? Ann Fr Anesth Reanim. 2014 Jul-Aug;33(7-8):453-6. https://doi.org/10.1016/j. annfar.2014.07.743
PMID:25168302

10. Vargas-Domínguez C, GochicoaRanfe M, Velásquez-Uncal $R$, Mejía-Alfaro M, Vásquez-García $J C$, Pérez-Padilla R, et al. Pruebas de función respiratoria, ¿cuál y a quién? Neumol Cir Torax. 2011;70(2):101-17.

11. Smetana GW, Lawrence VA, Cornell JE. Preoperative Pulmonary Risk Stratification for Noncardiothoracic Surgery: Systematic Review for the American College of Physicians. Annals of Internal Medicine [Internet]. American College of Physicians; 2006 Apr 18;144(8):581. http:// dx.doi.org/10.7326/0003-4819144-8-200604180-00009

12. Huh J, Sohn TS, Kim JK, Yoo YK, Kim DK. Is routine preoperative spirometry necessary in elderly patients undergoing laparoscopy-assisted gastrectomy? J Int Med Res. 2013 Aug;41(4):1301-9. https://doi. rg/10.1177/0300060513489470 PMID:23908552

13. Degani-Costa LH, Faresin SM, dos Reis Falcão LF. Preoperative evaluation of the patient with pulmonary disease. Braz J Anesthesiol. 2014 Jan-Feb;64(1):22-34. https://doi.org/10.1016/j.bja- 
ne.2012.11.002 PMID:24565385

14. Agarwal R, Porter M, Obeid G. Common Medical Illnesses that Affect Anesthesia and Their Anesthetic Management. Oral Maxillofacial Surg Clin N Am.; 2013. pp. 407-38.

15. Global strategy for the diagnosis, management, and prevention of chronic obstructive pulmonary disease (updated 2016). Global initiative for chronic obstructive lung disease: 2016.

16. Global strategy for asthma management and prevention. Global initiative for asthma: 2016.

17. Levett DZ, Grocott MP. Cardiopulmonary exercise testing for risk prediction in major abdominal surgery. Anesthesiol Clin. 2015 Mar;33(1):1-16. https://doi.org/10.1016/j. anclin.2014.11.001 PMID:25701925

18. Hennis PJ, Meale PM, Grocott MP. Cardiopulmonary exercise testing for the evaluation of perioperative risk in non-cardiopulmonary surgery. Postgrad Med J. 2011 Aug;87(1030):550-7. https://doi. org/10.1136/pgmj.2010.107185 PMID:21693573

19. Smith TB, Stonell $C$, Purkayastha S, Paraskevas P. Cardiopulmonary exercise testing as a risk assessment method in non cardio-pulmonary surgery: a systematic review. Anaesthesia. 2009 Aug;64(8):883-93. https://doi.org/10.1111/ j.1365-2044.2009.05983.x PMID:19604193

20. Paisani DM, Fiore JF Jr, Lunardi AC, Colluci DB, Santoro IL, Carvalho $C R$, et al. Preoperative 6-min walking distance does not predict pulmonary complications in upper abdominal surgery. Respirology. 2012 Aug;17(6):10137. https://doi.org/10.1111/ j.1440-1843.2012.02202.x PMID:22616954
21. Hightower $C E$, Riedel BJ, Feig BW, Morris GS, Ensor JE Jr, Woodruff VD, et al. A pilot study evaluating predictors of postoperative outcomes after major abdominal surgery: physiological capacity compared with the ASA physical status classification system. Br J Anaesth. 2010 Apr;104(4):465-71. https:// doi.org/10.1093/bja/aeq034 PMID:20190255

22. Ganai S, Ferguson MK. Can we predict morbidity and mortality before an operation? Thorac Surg Clin. 2013 Aug;23(3):28799. https://doi.org/10.1016/j. thorsurg.2013.04.001 PMID:23931013

23. Snowden CP, Prentis JM, Anderson HL, Roberts DR, Randles $D$, Renton $M$, et al. Submaximal cardiopulmonary exercise testing predicts complications and hospital length of stay in patients undergoing major elective surgery. Ann Surg. 2010 Mar;251(3):53541. https://doi.org/10.1097/ SLA.0b013e3181cf811d PMID:20134313

24. West MA, Lythgoe D, Barben CP, Noble L, Kemp GJ, Jack S, et al. Cardiopulmonary exercise variables are associated with postoperative morbidity after major colonic surgery: a prospective blinded observational study. $\mathrm{Br} J$ Anaesth. 2014 Apr;112(4):66571. https://doi.org/10.1093/bja/ aet408 PMID:24322573

25. West MA, Parry MG, Lythgoe D, Barben CP, Kemp GJ, Grocott $M P$, et al. Cardiopulmonary exercise testing for the prediction of morbidity risk after rectal cancer surgery. Br J Surg. 2014 Aug;101(9):1166-72. https:// doi.org/10.1002/bjs.9551 PMID:24916313

26. Hartley RA, Pichel AC, Grant SW, Hickey GL, Lancaster PS, Wisely NA, et al. Preoperative cardiopulmonary exercise tes- ting and risk of early mortality following abdominal aortic aneurysm repair. Br J Surg. 2012 Nov;99(11):1539-46. https:// doi.org/10.1002/bjs.8896 PMID:23001820

27. Moyes LH, McCaffer CJ, Carter RC, Fullarton GM, Mackay CK, Forshaw MJ. Cardiopulmonary exercise testing as a predictor of complications in oesophagogastric cancer surgery. Ann R Coll Surg Engl. 2013 Mar;95(2):12530. https://doi.org/10.1308/ rcsann.2013.95.2.125 PMID:23484995

28. Carlisle J, Swart M. Mid-term survival after abdominal aortic aneurysm surgery predicted by cardiopulmonary exercise testing. Br J Surg. 2007 Aug;94(8):966-9. https://doi.org/10.1002/bjs.5734 PMID:17440956

29. Zraier $S$, Haouache H, Dhonneur $\mathrm{G}$. Which preoperative respiratory evaluation? Ann Fr Anesth Reanim. 2014 Jul-Aug;33(7-8):453-6. https://doi.org/10.1016/j. annfar.2014.07.743

PMID:25168302

30. Masoomi H, Reavis KM, Smith BR, Kim H, Stamos MJ, Nguyen NT. Risk factors for acute respiratory failure in bariatric surgery: data from the Nationwide Inpatient Sample, 2006-2008. Surg Obes Relat Dis. 2013 Mar-Apr;9(2):277-81. https://doi.org/10.1016/j. soard.2012.01.025 PMID:22534604

31. Clavellina-Gaytán $D$, VelázquezFernández D, Del-Villar E, Domínguez-Cherit G, Sánchez $\mathrm{H}$, Mosti M, et al. Evaluation of spirometric testing as a routine preoperative assessment in patients undergoing bariatric surgery. Obes Surg. 2015 Mar;25(3):530-6. https://doi. org/10.1007/s11695-014-1420-x PMID:25240391 
32. Lo IL, Siu CW, Tse HF, Lau TW, Leung F, Wong M. Preoperative pulmonary assessment for patients with hip fracture. Osteoporos Int. 2010 Dec;21(S4 Suppl 4):S579-86. https://doi. org/10.1007/s00198-010-14277 PMID:21057997

33. Société de pneumologie de langue francaise. Recommandations pour la pratique clinique concernant les explorations fonctionnelles respiratoires. Rev Mal Respir. 2011;28:1183-92.

34. Al-Ruzzeh S, Kurup V. Respiratory diseases. In: Hines RL, Marschall KE, editors. Stoelting's anesthesia and co-existing disease. 6th ed. Philadelphia: Elsevier Saunders; 2012. pp. 181-217.

35. Dones F, Foresta G, Russotto V. Update on perioperative management of the child with asthma. Pediatr Rep. 2012 Apr;4(2):e19. https://doi.org/10.4081/pr.2012. e19 PMID:22802997

36. Becker DE. Preoperative medical evaluation: part 2: pulmonary, endocrine, renal, and miscellaneous considerations. Anesth Prog. 2009;56(4):135-44. https:// doi.org/10.2344/0003-300656.4.135 PMID:20020794

37. Al-Ruzzeh S, Kurup V. Respiratory diseases. In: Hines RL, Marschall KE, editors. Stoelting's anesthesiaand co-existing disease. 6th ed. Philadelphia: Elsevier Saunders; 2012. pp. 181-217.

38. Levett DZ, Grocott MP. Cardiopulmonary exercise testing for risk prediction in major abdominal surgery. Anesthesiol Clin. 2015 Mar;33(1):116. https://doi.org/10.1016/j. anclin.2014.11.001 PMID:25701925

39. Smetana GW. S metana GW. Postoperative pulmonary complications: an update on risk assessment and reduction. Cleve Clin J Med. 2009;76 suppl 4:S60-5. https://doi.org/10.3949/ ccjm.76.s4.10.

40. Shander A, Fleisher LA, Barie PS, Bigatello LM, Sladen RN, Watson CB. Clinical and economic burden of postoperative pulmonary complications: patient safety summit on definition, risk-reducing interventions, and preventive strategies. Crit Care Med. 2011 Sep;39(9):216372. https://doi.org/10.1097/ cCM.0b013e31821f0522 PMID:21572323

41. Canet J, Gallart L, Gomar C, Paluzie G, Vallès J, Castillo J, et al.; ARISCAT Group. Prediction of postoperative pulmonary complications in a populationbased surgical cohort. Anesthesiology. 2010 Dec;113(6):133850. https://doi.org/10.1097/ ALN.0b013e3181fc6e0a PMID:21045639

41. American Society of Anesthesiologist. ASA Physical Status Classification System. 2014. Disponible en: https://www.asahq.org/resources/ clinical-information/asa-physicalstatus-classification-system

42. Treschan TA, Malbouisson LM, Beiderlinden M. Intraoperative mechanical ventilation strategies to prevent postoperative pulmonary complications in patients with pulmonary and extrapulmonary comorbidities. Best Pract Res Clin Anaesthesiol. 2015 Sep;29(3):341-55. https://doi. org/10.1016/j.bpa.2015.08.002 PMID:26643099

43. Canet J, Gallart L, Gomar C, Paluzie G, Vallès J, Castillo J, et al.; ARISCAT Group. Prediction of postoperative pulmonary complications in a populationbased surgical cohort. Anesthesiology. 2010 Dec;113(6):133850. https://doi.org/10.1097/ ALN.0b013e3181fc6e0a PMID:21045639

44. Mazo V, Sabaté S, Canet J, Gallart L, de Abreu MG, Belda J, et al. Prospective external validation of a predictive score for postoperative pulmonary complications. Anesthesiology. 2014 Aug;121(2):21931. https://doi.org/10.1097/ ALN.0000000000000334 PMID:24901240

45. Arozullah AM, Khuri SF, Henderson WG, Daley J; Participants in the National Veterans Affairs Surgical Quality Improvement Program. Development and validation of a multifactorial risk index for predicting postoperative pneumonia after major noncardiac surgery. Ann Intern Med. 2001 Nov;135(10):847-57. https://doi.org/10.7326/00034819-135-10-200111200-00005 PMID:11712875

46. Johnson RG, Arozullah AM, Neumayer L, Henderson WG, Hosokawa P, Khuri SF. Multivariable predictors of postoperative respiratory failure after general and vascular surgery: results from the patient safety in surgery study. J Am Coll Surg. 2007 Jun;204(6):118898. https://doi.org/10.1016/j. jamcollsurg.2007.02.070 PMID:17544077

47. Cleva R, Assumpção MS, Sasaya F, Chaves NZ, Santo MA, Fló $C$, et al. Correlation between intra-abdominal pressure and pulmonary volumes after superior and inferior abdominal surgery. Clinics (São Paulo). 2014 Jul;69(7):483-6. https://doi. org/10.6061/clinics/2014(07)07 PMID:25029580

48. Bablekos GD, Michaelides SA, Analitis A, Charalabopoulos KA. Effects of laparoscopic cholecystectomy on lung function: a systematic review. World J Gastroenterol. 2014 Dec;20(46):17603-17. https:// doi.org/10.3748/wjg.v20. i46.17603 PMID:25516676

49. Oh TK, Park IS, Ji E, Na H-S. Value of preoperative spirometry 
test in predicting postoperative pulmonary complications in high-risk patients after laparoscopic abdominal surgery. Subramaniam B, editor. PLOS ONE [Internet]. Public Library of Science (PLoS); 2018 Dec 19;13(12):e0209347. Available from: http://dx.doi.org/10.1371/ journal.pone.0209347

50. Huh J, Sohn TS, Kim JK, Yoo YK, Kim DK. Is routine preoperative spirometry necessary in elderly patients undergoing laparoscopy-assisted gastrectomy? J Int Med Res. 2013 Aug;41(4):1301-9. https://doi. rg/10.1177/0300060513489470

\section{PMID:23908552}

51. Lo IL, Siu CW, Tse HF, Lau TW, Leung F, Wong M. Preoperative pulmonary assessment for patients with hip fracture. Osteoporos Int. 2010 Dec;21(S4 Suppl 4):S579-86. https://doi. org/10.1007/s00198-010-14277 PMID:21057997

52. Lao L, Weng X, Qiu G, Shen J. The role of preoperative pulmonary function tests in the surgical treatment of extremely severe scoliosis. J Orthop Surg Res. 2013 Sep;8(1):32. https://doi. org/10.1186/1749-799X-8-32 PMID:24007407

53. Keeratichananont W, Thanadet- suntorn C, Keeratichananont S. Value of preoperative 6-minute walk test for predicting postoperative pulmonary complications. Ther Adv Respir Dis. 2016 Feb;10(1):18-25. https://doi. rg/10.1177/1753465815615509 PMID:26546478

54. Magalhães CB, Nogueira IC, Marinho LS, Daher EF, Garcia $J \mathrm{H}$, Viana CF, et al. Exercise Capacity Impairment Can Predict Postoperative Pulmonary Complications after Liver Transplantation. Respiration. 2017;94(3):272-8. https:// doi.org/10.1159/000479008 PMID:28738386 